\title{
Electron Accepting Benzodithiophene-4,8-dicarboxylate Designed for New Type D-A Narrow Bandgap Copolymers
}

\author{
Kosuke Shibasaki ${ }^{1, a}$ and Masashi Kijima ${ }^{2,3, b, ~}{ }^{*}$ \\ ${ }^{1}$ Institute of Materials Science, Graduate School of Pure and Applied Science, University of Tsukuba, \\ 1-1-1 Tennodai, Tsukuba, Ibaraki 305-8573, Japan. \\ ${ }^{2}$ Tsukuba Research Center for Interdisciplinary Materials Science, University of Tsukuba, 1-1-1 \\ Tennodai, Tsukuba, Ibaraki 305-8573, Japan. \\ ${ }^{3}$ Division of Materials Science, Faculty of Pure and Applied Science, University of Tsukuba, 1-1-1 \\ Tennodai, Tsukuba, Ibaraki 305-8573, Japan. \\ E-mail: ${ }^{a}$ S-shibasaki@ims.tsukuba.ac.jp, ${ }^{b}$ kijima@ims.tsukuba.ac.jp
}

Keywords: benzodithiophene, thienothiazole, donor-acceptor conjugated polymer

\begin{abstract}
An acceptor (A) unit of benzodithiophene-4,8-dicarboxylate (BDTC) was combined with a donor (D) unit of thieno(3,4- $d$ ) thiazole (TTz) to synthesize a D-A type conjugated polymer, PBDTC-TTz. Bandgap of PBDTC-TTz was narrow, estimated to be $1.57 \mathrm{eV}$. The $\pi$-stacking distance observed by XRD was small value to be $3.55 \AA$. The energy level of the highest occupied molecular orbital was estimated to be $-5.03 \mathrm{eV}$, which was deeper than that another D-A type copolymer composed of BDTC and thieno(3,4-b)thiophene, previously synthesized.
\end{abstract}

\section{Introduction}

Recently, we have developed a new type donor-acceptor (D-A) polymer, poly[4,8-benzo(1,2-b:4,5-b')dithiophenedicarboxylate-alt-thieno(3,4-b)thiophene] (PBDTC-TT) composed of an electron accepting benzodithiophene-4,8-dicarboxylate (BDTC) and an electron donating thieno(3,4-b)thiophene (TT), as shown in Figure 1. ${ }^{[1]}$ PBDTC-TT exhibited narrow bandgap nature $(1.5 \mathrm{eV})$ and broad photoabsorption owing to strong intermolecular charge transfer (ICT) between the BDTC and the TT units. The bulk heterojunction (BHJ) organic photovoltaic cell (OPV) based on PBDTC-TT showed the power conversion efficiency (PCE) of 3.0\% and efficient sensitization in the range of visible light and near infrared as far as $900 \mathrm{~nm}$. These results indicated that the BDTC unit is a promising A-type building block to construct narrow bandgap copolymers. However, PBDTC-TT was revealed to have shallow energy level of the highest occupied molecular orbital $\left(E_{\mathrm{HOMO}}\right)(-4.80$ $\mathrm{eV})$, which would limit further improvement of PCE on OPV.

In this work, BDTC was combined with thieno(3,4-d)thiazole (TTz) that has been known to possess weaker electron donating ability than that of TT, constructing the D-A type copolymer, poly[4,8-benzo(1,2-b:4,5- $b$ ')dithiophene dicarboxylate-alt-thieno(3,4-d)thiazole] (PBDTC-TTz), as shown in Figure 1. The effect of the electron donating $\mathbf{T T z}$ on $E_{\mathrm{HOMO}}$ of the BDTC based polymers was investigated in order to achieve narrow bandgap nature and deep $E_{\mathrm{HOMO}}$ for OPVs.

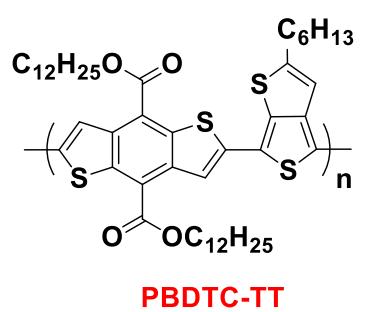

PBDTC-TT

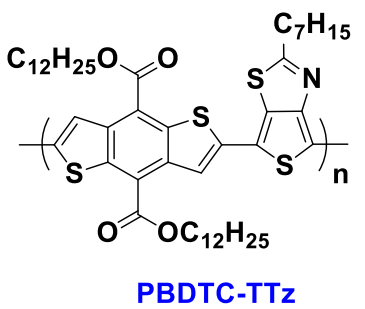

PBDTC-TTz

Figure 1. Chemical structures of $\pi$-conjugated alternating copolymers, PBDTC-TT and PBDTC-TTz. 


\section{Experimental}

\subsection{Measurements}

${ }^{1} \mathrm{H}$ NMR spectrum was obtained with a JEOL JNM-ECS 400 at resonance frequency of $400 \mathrm{MHz}$ for ${ }^{1} \mathrm{H}$ in $\mathrm{CDCl}_{3}$. The number average molecular weight $\left(M_{\mathrm{n}}\right)$, weight average molecular weight $\left(M_{\mathrm{w}}\right)$ and polydispersity index (PDI) of soluble fraction of the polymers in chloroform were determined by gel permeation chromatography (GPC) calibrated with polystyrene standards using chloroform as an eluent at room temperature. Ultraviolet-visible (UV-vis) spectra of the polymers were measured on a Shimadzu UV-1800 spectrophotometer. Cyclic voltammetry (CV) of the polymers in a thin film state on Pt was carried out in $0.1 \mathrm{MEt}_{4} \mathrm{NBF}_{4}$ acetonitrile solution at a scan rate of $50 \mathrm{mV} \mathrm{s}^{-1}$ under an argon atmosphere by using a Hokuto Denko HB-305 function generator and a HAL3001 potentiostat equipped with a Pt inlay-disk as the working electrode, a Pt plate as the counter electrode, and a saturated calomel electrode (SCE) as the reference electrode. Ionization potentials (vs vacuum) of the polymers were estimated from the onset of their first oxidation peak $\left(E_{\mathrm{a}}{ }^{\text {onset }}\right)$ in the cyclic voltammograms on the basis that ferrocene / ferrocenium is $4.8 \mathrm{eV}$ below the vacuum level. ${ }^{[2]} \mathrm{X}$-ray diffraction (XRD) measurements of drop-cast polymer films were carried out using a Rigaku MiniFlex600.

\subsection{Materials}

All chemicals were purchased from Kanto Chemical Co., Tokyo Chemical Industry Co., Nacalai Tesque, or Sigma-Aldrich, LLC., and they were used without further purification unless stated. Tetrahydrofuran (THF) was purified by distillation according to common method. 2,6-Dibromo-4,8-benzodithiophenedicarboxylate $\left(\mathbf{B D T C}-\mathbf{B r}_{2}\right)$ and 2-heptylthieno(3,4-d)thiazole were synthesized according to literature methods. ${ }^{[1,3]}$

Synthesis of PBDTC-TTz. In a two-necked flask, BDTC-Br 2 (80.0 $\mathrm{mg}, 0.104 \mathrm{mmol})$, 2-heptylthieno(3,4- $d$ ) thiazole $(24.7 \mathrm{mg}, 0.104 \mathrm{mmol})$, dry THF $(1.04 \mathrm{ml})$, tris(dibenzylideneacetone)dipalladium $(0)$-chloroform adduct $\left(\mathrm{Pd}_{2}\left(\mathrm{dba}_{3} \cdot \mathrm{CHCl}_{3}\right)(5.38 \mathrm{mg})\right.$, tris(2-methoxyphenyl)phosphine $\left(\mathrm{P}(\mathrm{o}-\mathrm{MeOPh})_{3}(7.30 \mathrm{mg})\right.$, pivalic acid $(\mathrm{PivOH})(10.6 \mathrm{mg})$ and cesium carbonate $\left(\mathrm{Cs}_{2} \mathrm{CO}_{3}\right)$ $(101.1 \mathrm{mg})$ were mixed with stirring at room temperature. After stirring for $30 \mathrm{~min}$, the reaction mixture was heated at $80{ }^{\circ} \mathrm{C}$ for $1 \mathrm{~h}$. After cooling to room temperature, the polymeric products were precipitated from methanol. The precipitates were successively extracted with methanol, acetone, hexane and chloroform by Soxhlet extraction. The chloroform extract was again precipitated from methanol. PBDTC-TTz was obtained as a black solid (41 mg, 46\% yield). ${ }^{1} \mathrm{H}$ NMR (400 MHz, $\mathrm{CDCl}_{3}, \delta / \mathrm{ppm}$ ) 0.89-2.50 (br, 59H), 3.07 (br, 2H), 4.64 (br, 4H), 7.69 (br, 2H). Anal. Calcd for $\mathrm{C}_{48} \mathrm{H}_{69} \mathrm{NO}_{4} \mathrm{~S}_{4}: \mathrm{C}, 67.64 ; \mathrm{H}, 8.16 ; \mathrm{N}, 1.64$. Found: $\mathrm{C}, 66.83 ; \mathrm{H}, 7.50 ; \mathrm{N}, 1.72$.

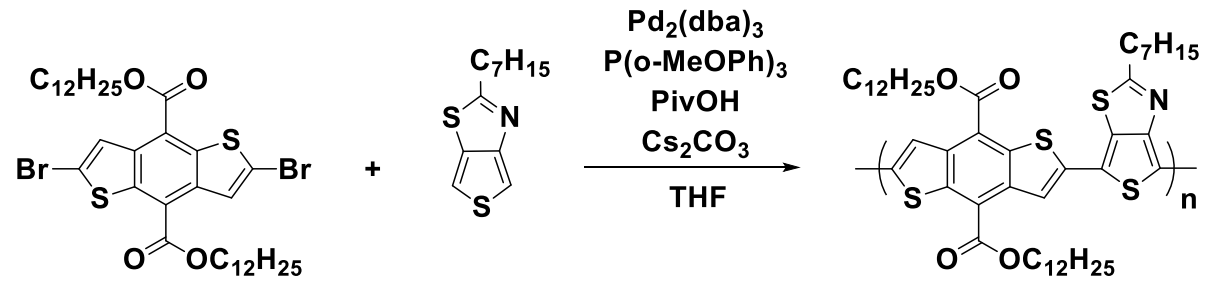

Scheme 1. Synthesis of PBDTC-TTz. PivOH = pivalic acid, THF = tetrahydrofuran.

\section{Results and discussion}

\subsection{Synthesis and optical properties}

A synthetic route for PBDTC-TTz is shown in Scheme 1. PBDTC-TTz was obtained by direct arylation polymerization according to literature methods. ${ }^{[4]}$ PBDTC-TTz was highly soluble in common organic solvents such as dichloromethane and chloroform. $M_{\mathrm{n}}$ and PDI were $7.4 \mathrm{~kg} \mathrm{~mol}^{-1}$ and 1.9 , respectively. 
Figure 2 shows the absorption spectrum of PBDTC-TTz with that of PBDTC-TT as a reference. The results are summarized in Table 1. The absorption maximum $\left(\lambda_{\max }\right)$ of PBDTC-TTz in chloroform was observed at $659 \mathrm{~nm}$, which was blue-shifted compared with that of PBDTC-TT $\left(\lambda_{\max }\right.$ $=702 \mathrm{~nm}$ ). The blue shift is due to weak ICT in the PBDTC-TTz, which could be interpreted that electron donating ability of $\mathbf{T T z}$ is lower than that of TT. In the film state, PBDTC-TTz exhibited two absorption peaks with $\lambda_{\max }$ at 660 and $716 \mathrm{~nm}$, and the absorption onset $\left(\lambda_{\text {onset }}\right)$ was almost the same as that in solution. The optical bandgap $\left(E_{\mathrm{g}}{ }^{\text {opt }}\right)$ of PBDTC-TTz determined from $\lambda_{\text {onset }}$ of the film state was $1.57 \mathrm{eV}$, which was wider than that of PBDTC-TT $\left(E_{\mathrm{g}}{ }^{\mathrm{opt}}=1.50 \mathrm{eV}\right)$ but spectral width is much broader than that of the prototype D-A copolymer, PBDTTTz-1 composed of the D unit of 4,8-dialkoxybenzodithiophene (BDT) and the A unit of $\mathbf{T T z}\left(\lambda_{\max }=625 \mathrm{~nm}, E_{\mathrm{g}}{ }^{\mathrm{opt}}=1.8 \mathrm{eV}\right)^{[5]}$. ICT interaction in the combination of D-type TTz and A-type BDTC might be stronger than that in the combination of A-type TTz and D-type BDT.
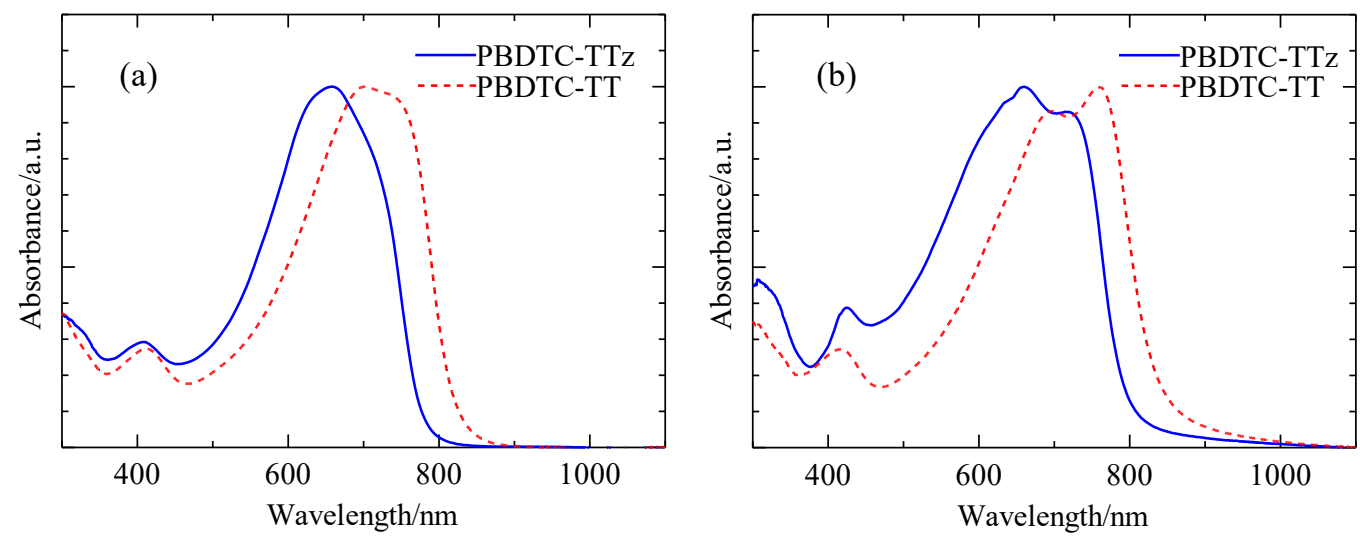

Figure 2. UV-vis absorption spectra of PBDTC-TTz and PBDTC-TT (a) in chloroform solution and (b) in the film state.

Table 1. Optical properties and energy levels of PBDTC-TTz and PBDTC-TT.

\begin{tabular}{|c|c|c|c|c|}
\hline \multirow[b]{2}{*}{ Polymer } & \multicolumn{2}{|c|}{ UV-vis absorption } & \multirow[b]{2}{*}{$\begin{array}{c}E_{\mathrm{HOMO}} \\
(\mathrm{eV})\end{array}$} & \multirow[b]{2}{*}{$\begin{array}{c}E_{\mathrm{g}}^{\text {opt d }} \\
(\mathrm{eV})\end{array}$} \\
\hline & $\begin{array}{c}\text { Solution }^{\mathrm{a}} \\
\lambda_{\max } \\
(\mathrm{nm})\end{array}$ & $\begin{array}{c}\text { Film } \\
\lambda_{\max } \\
(\mathrm{nm})\end{array}$ & & \\
\hline PBDTC-TTz & 659 & 660,716 & $-5.03^{\mathrm{b}}$ & 1.57 \\
\hline PBDTC-TT & 702 & 707,757 & $-4.88^{\mathrm{b}}(-4.80)^{\mathrm{c}}$ & 1.50 \\
\hline
\end{tabular}

\footnotetext{
${ }^{\mathrm{a}}$ Measured in $\mathrm{CHCl}_{3}$.

${ }^{\mathrm{b}}$ Estimated with $\mathrm{CV}$

${ }^{\mathrm{c}}$ Estimated with photoelectron spectroscopy in the atmosphere.

${ }^{\mathrm{d}}$ Estimated from the absorption band edge of the films.
}

\subsection{Electrochemical properties}

Cyclic voltammogram of PBDTC-TTz and PBDTC-TT are shown in Figure 3 . The $E_{\mathrm{HOMO}}$ determined by cyclic voltammetry are summarized in Table 1. PBDTC-TTz and PBDTC-TT exhibited quasi-reversible redox waves. The $E_{\text {HOMO }}$ of PBDTC-TTz estimated from the onset oxidation potential was to be ca. $-5.03 \mathrm{eV}\left(E_{\mathrm{a}}{ }^{\text {onset }}=0.66 \mathrm{~V}\right)$, which was deeper than that of PBDTC-TT $\left(E_{\mathrm{HOMO}}=-4.88 \mathrm{eV}, E_{\mathrm{a}}{ }^{\text {onset }}=0.51 \mathrm{~V}\right)$. 


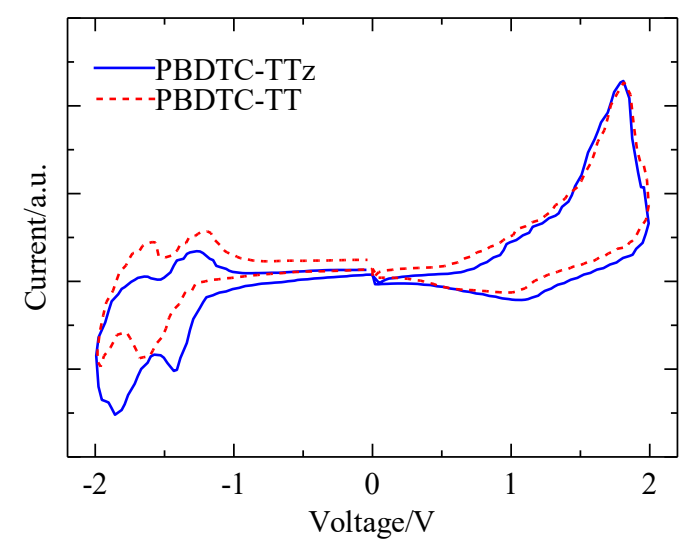

Figure 3. Cyclic voltammograms of PBDTC-TTz and PBDTC-TT.

\subsection{X-ray diffraction analysis}

XRD analyses were performed to investigate the structural order of the polymers in the film state. PBDTC-TTz showed two diffraction peaks at $2 \theta=3.64^{\circ}$ and $25.1^{\circ}\left(d_{1}=24.3 \AA, d_{2}=3.55 \AA\right)$, which are almost the same as those of PBDTC-TT $\left(d_{1}=24.7 \AA, d_{2}=3.57 \AA\right)$ as shown in Figure 4 . The former diffractions correspond to the interchain distance between the polymer backbones separated by the side chains, while the latter peaks correspond to the $\pi$-stacking distance between the planar polymer backbones. These results indicate that introduction of TTz could deepen $E_{\mathrm{HOMO}}$ of BDTC based polymer without increase of the interchain distance and $\pi$-stacking distance.

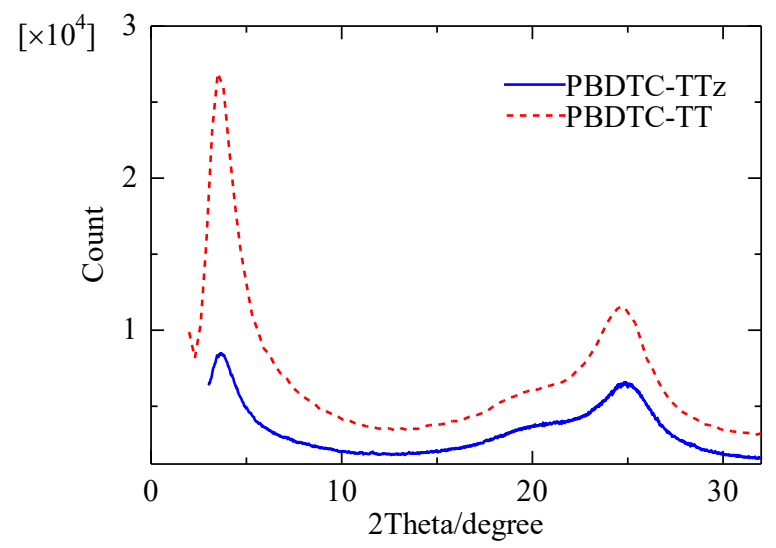

Figure 4. X-ray diffraction patterns of the drop cast films of the polymers.

\section{Conclusions}

An alternating D-A type copolymer PBDTC-TTz composed of BDTC and TTz was synthesized and characterized. PBDTC-TTz exhibited wider $E_{\mathrm{g}}$ compared to PBDTC-TT due to weaker ICT effect between BDTC and TTz, but its $E_{\mathrm{g}}$ was sufficiently narrow to be applicable to OPVs. Incorporation of $\mathbf{T T z}$ in place of $\mathbf{T T}$ lowered $E_{\mathrm{HOMO}}$ of the polymer. The polymer structure of PBDTC-TTz evaluated XRD analysis was comparable to that of PBDTC-TT. These preliminary studies indicated that $E_{\mathrm{HOMO}}$ of BDTC based polymer could be lowered, which is derived from the weak electron-donating unit with the narrow bandgap nature. Further studies to investigate the effect of the TTz unit on the BHJ OPV performance currently are under way. 


\section{Acknowledgments}

This work was partially supported by Tsukuba Nanotechnology Human Resource Development Program, University of Tsukuba, and we thank a guest Prof. Dr. N. Ota, University of Tsukuba, a guest Prof. Dr. M. Kitajima, University of Tsukuba, and a guest Prof. Dr. T. Yasuda, National Institute for Material Science and University of Tsukuba (TIMS), for helpful discussion through the program. We thank Prof. Dr. Y. Yamamoto, University of Tsukuba, for XRD measurement. We thank Chemical Analysis Division, Research Facility Center for Science and Technology, University of Tsukuba, for facilities of the NMR and elemental analysis.

\section{References}

[1] K. Shibasaki, K. Tabata, Y. Yamamoto, Y. Yasuda, M. Kijima, Macromolecules 47 (2014) 4987-4993.

[2] A. K. Agrawal, S. A. Jenekhe, Chem. Mater. 8 (1996) 579-589.

[3] J. W. Jo, S. S. Kim, W. H. Jo, Org. Electron. 13 (2012) 1322-1328.

[4] P. D. Homyak, J. Tinkham, P. M. Lahti, E. B. Coughlin, Macromolecules 46 (2013) 8873-8881.

[5] N. Allard, S. Beaupré, B. R. Aïch, A. Najari, Y. Tao, M. Leclerc, Macromolecules 44 (2011) 7184-7187. 